\title{
Innovative policy instruments for urban governance: the case of urban regeneration in the Danube Region
}

\author{
Barbara MUŠIČ, Urban Planning Institute of the Republic of Slovenia \\ Matej NIKŠIČ, Urban Planning Institute of the Republic of Slovenia \\ Andrada LUPULESCU, Urbasofia, Romania \\ Emilia BUDAU, Urbasofia, Romania \\ Serin GEAMBAZU, urban planning expert, Romania
}

\begin{abstract}
Urban regeneration is gaining the importance within the sustainable development paradigm in which achieving the better efficiency of the already urbanised areas shall be given a paramount priority before the spread of the city into the new territories. The urban areas that are underused or abandoned bear a high potential in these terms. However, such areas are rather complex to handle due to their legal status, ownership structures, financial issues, state of the art of the physical and functional infrastructures, stakeholders' interests and not the least sectoral regulations and policies. The local public administration is often confronted with the several ramifications when making the regeneration processes happen, while at the same time may lack the basic insights, knowledge and tools to most efficiently lead these processes. This paper presents partial results of the AGORA project that aims to rise the capacity of public administrations in the European Danube Region in their inner-city urban regeneration endeavours. Through various project activities it supports local planning authorities to develop their own Urban Regeneration Agendas. A part of the capacity building within the project is the exchange of good practices among partners, among others the innovative policy instruments that different AGORA partners already use in the urban regeneration processes. The paper presents which tools are already used by the project partners and analyses them from the points of view of their administrative status, typology regarding the main goals they address, their legal status and the level of inclusiveness.
\end{abstract}

\section{Keywords}

public administration, innovative policy instruments, inner city, urban regeneration, European Danube Region 


\section{Introduction}

Consequences of deindustrialisation, urban transition and changing living patterns left (especially) small and middle size cities struggling to remain unattractive and uncompetitive compared to other cities in the Danube Region. New urban planning and management approaches are needed to unlock the potentials these cities possess. On one side there is still a trend of the centralised approach of the governance system, where the higher-tier governments hold critical power to make decisions affecting the local level. On the other side different stakeholders and the civil society has been activated too and organised itself in order to improve the urban vitality and quality of urban life itself. Both approaches - top-down and bottom-up - are much needed to improve the vitality and image of mid and small sized cities of the Danube Region which are facing the raising number of abandoned areas. Public administration governance needs to go beyond traditional approaches in a way of developing new governance models which could support and speed up urban development processes. The development and implementation of innovative policy instruments play an important role in these endeavours.

In order to compare the existing state of the art in different parts of the Danube Region and seek the appropriate approaches to address challenges, the AGORA project has been initiated within the Interreg Danube Region Programme. The main aim of the AGORA is to achieve a substantial change in the process of the workflow of the public administration on the local and regional level through empowerment of their capacities (see http://www.interreg-danube.eu/approvedprojects/agora for more information). The intention of the AGORA project is to help public administrations becoming a credible and active leaders of urban regeneration processes through codesigning and testing new governance models and policy instruments on underused and unused urban areas of the inner cities. 10 partnering localities from the Danube Region are co-creating new venues for stakeholders and public involvement (so called AGORA Communities) which will through actions and workshops develop 10 AGORA Urban Regeneration Agendas (URAs) and 10 concrete pilot actions. Based on the AGORA experience, the policy-learning platform is being developed to support cities to reintegrate and reconnect local undervalued areas and though new social and economic activities improve their value.

Prerequisite for a successful co-designing of urban development strategies is to get acquainted with the state of the art of the governance models and decision-making procedures, to recognise the spatial potentials in the cities as well as to recognise available policy instruments that can help achieving the urban (re)development goals by involving all relevant stakeholders into the codesigning processes - involving stakeholders into the process of finding the room for improvements is seen as one of the crucial conditions for a long-term success.

As a part of the AGORA methodology, cities have to prepare so called operational analyses as a starting point to define specific objectives which they would like to follow in the process of codesigning their Urban regeneration Agendas (URAs). In the operational analyses they consider their existing governance systems, available policy instruments, identify potential stakeholders and identify and analyse underused and/or unused urban areas as local potentials for urban regeneration. 10 territorial partners from different countries with different history and cultural background have different objectives, but since they are coming from the same macro-region, there are similarities among them too. The framework of trans-national AGORA project allows un-locking of potentials and building-up the opportunities via exchanges of good practices, peer-to-peer exchanges and learning sessions. 
This paper presents a minor part of reach contents of the AGORA project - the innovative policy instruments that partnering cities have already started to use in order to overcome the obstacles of rather rigid planning systems and procedures and to kick-off and/or accelerate the urban regeneration processes in a collaborative manner. The innovative instruments are usually used to provide a more flexible planning environment that can better address the fast-changing circumstances and needs of the spatial, economic and social fabrics of the Danube Region's small and medium sized cities. These innovative policy instruments are presented through different aspects that need to be taken into consideration when one aims to transfer them from one successful example to other environments, among others:

- administrative level they address,

- their legal status (binding-nonbinding),

- basic objectives and aims (partnering/networking, management, financial/economic empowerment, information gathering/sharing, capacity building etc.),

- the level of inclusiveness they provide and

- the types of actors they involve, as well as the outcomes they manage to reach.

The review shows the variety of instruments that cities are using to improve their performance in the field of urban regeneration.

The concluding part of the paper highlights the innovative aspect that is present in the field of policy instruments which are used to accelerate the urban regeneration processes. It also points out the importance of the cooperation between the cities on the international level by illustrating how the AGORA project helps cities to understand their own ways of operating, compares themselves towards the others and make them to learn from each other by sharing good practices not only at the declarative levels but through the in-depth analytical processes that help to understand which tools can (not) be used in various contexts.

\section{Innovative governance instruments}

According to Howlett and Rayner (2007), the governance instruments are the "techniques of governance, which, one way or another, involve the utilization of state resources, or their conscious limitation, in order to achieve policy goals". As Schneider and Ingram (1990) put it, governance instruments are targeted "to get people do things that they might not otherwise do; or it enables people to do things that they might otherwise not have done".

Good governance is not based on the narrow selection of instruments but requires the authorities and other stakeholders to use the mix of instruments in order to overarch the goals or objectives to which the application of these instruments contribute. Such an ability of combining different tools can be innovative in order to adjust them to the needs of concrete case and also abilities of local stakeholders to provide best out-puts that will be best tailored to the local context. As such, governance instruments (and their locally-adjusted combinations) form the operational tools of public policy (Majoor and Schwartz, 2015).

There seems to be an agreement within the scholarly debates that there are two dominant approaches towards analysing, designing, and using governance instruments. The functionalist or instrumentalist approach sees instruments (or a mix of instruments) as rational, neutral, pragmatic tools, aimed at achieving certain specified goals and solving problems, while the political sociology approach stresses that instruments are not neutral (purely technical) tools, but embody a particular relationship between government and citizen (Lascoumes and Le Gales 2007). This observation stresses the need for the contextual understanding of the governance instruments - they are embedded within a particular socio- 
political and geographical context (so called place) reflected in a particular society-authority relationship and ideological foundation, which must always be taken into account when considering them. This was an important starting point when setting up the Agora project methodologies.

In order to address in the proper way, the complex socio-economic, functional and other challenges that define any urban regeneration case, the governance instruments need to be innovative. This relates to the ability to introduce a new product, a new idea, a new technology, or a new solution to the existing problem exists due to an inability of the classical approaches to problem-solving - as such, innovation may include only basic upgrading or a new combination of the existing, already known approaches, but can also be revolutionary and include the invention of completely new products, technologies, procedures etc. (Cirera et al, 2020). Generally speaking, the important part of any innovation policy is an overarching strategy with clearly defined end goals and can be delivered through a number of instruments that are used at once - thus, innovation policy consists of the combination of existing and new instruments that complement one another and are in a direct interaction - s.c. policy mix (Edquist, 2011; Freeman, 1987).

\section{Methodology}

One of the aims of AGORA project was to understand which innovative instruments of the governance the partnering cities are using in their urban regeneration endeavours. Therefore, the data on innovative instruments were collected through a common questionnaire developed by the research team of the AGORA project under the leadership of the Urban Planning Institute of the Republic of Slovenia. The questionnaire had multiple sections and served also for the collection of the other data needed for the project activities. Among others the partners were asked to analyse the following issues:

- the general administrative structure in their country, which helped in understanding the basic institutional structure of the state within which the urban planning apparatus is embedded,

- the local/regional government structure and their roles to reveal the responsibilities and power distribution within their administrative bodies,

- the decision-making process in the spatial planning, which pointed out the main actors and their roles in urban development in general and urban regeneration in particular, and

- policy instruments and governance models to look beyond the institutions of the local administration and to search for processes and mechanisms through which significant and resource-full actors coordinate their actions and resources in the pursuit of collectively defined objectives.

Within the last section of the questionnaire 10 AGORA territorial partners had to analyse the present innovative policy instruments in the field of urban regeneration, particularly those instruments that allow the underused or abandoned urban areas or plots to be re-activated faster and in a more collaborative and meaningful way. The starting assumption of these analyses was that for the support of successful and efficient implementation of urban regeneration projects, cities and regions must have developed other policy instruments and approaches aside the ones which are a part of the usual, legally binding urban planning and related procedures. Since the AGORA project is focused on building capacities of the AGORA public administration, this section was devoted to analysis of innovative policy instruments and governance models as creative solutions.

Innovative policy instruments were analysed from the following aspects:

- administrative level,

- type of policy instrument,

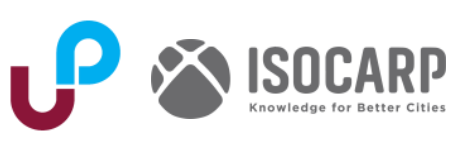


- legal status and

- type of the citizen participation the instrument seeks/allows for, namely the way the instrument intends/manages to embed the public participation.

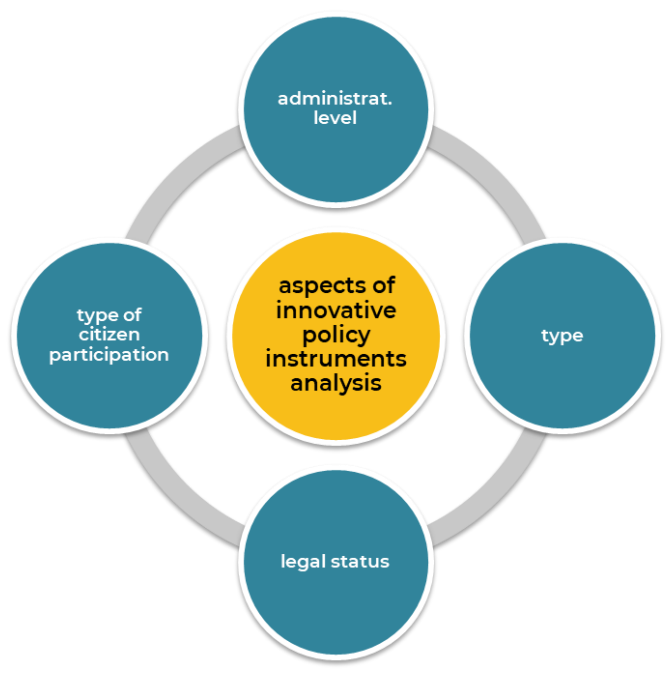

Figure 1 - Aspects of innovative policy instruments analysis. Source: UIRS.

10 Agora territorial partners contributed the data, 8 from the city level, i.e. Chisinau (Moldova), ClujNapoca (Romania), Koprivnica and Slavonski Brod (Croatia), Kranj (Slovenia), Sofia (Bulgaria), Szarvas (Hungary) and Zenica (Bosnia and Herzegovina)), 1 from the regional level, i.e. Neckar-Alb Region (Germany) and 1 from the district level, Prague 9 (Czech Republic). Each partner had to contribute at least 3 innovative policy instruments, altogether 35 innovative policy instruments were listed and analysed.

\section{Results}

The data contributed by the partners shows that the urban regeneration is an important aspect contributing to sustainable development goals which cities are following in the process of their development. Since spatial and master planning policy instruments are binding documents, guidelines which are addressing urban regeneration are very important in the process of co-creating the Urban Regeneration Agendas (URAs). As the legal planning policy instruments to often are not efficiently supporting the successful implementation of city programmes and projects, cities in addition developed other planning policy instruments, e.g. Strategies for urban regeneration, Integrated Urban Strategies for Sustainable Development, etc. These instruments have been developed for different purposes (e.g. applying different founds, supporting the development of spatial development plans, decision making processes, etc.). Aside from these instruments cities also developed other policy instruments which are very creative and innovative in their efforts to find solutions which are supporting the effective governance and implementation of projects in their territories.

The data related to innovative policy instruments were collected and analysed based on the input from 10 operational analysis reports which 10 AGORA territorial partners had to prepare in line with the project framework and not based on the scientific research. The scientific partners of the AGORA under the coordination of the Urban Planning Institute of the Republic of Slovenia developed the methodology and provide guidelines which cities needed to follow in the phase of preparing their state-of-the-art analysis. The main aim was to identify and classify innovative policy instruments which they are already using or seeing them as potentially useful in the urban regeneration interventions in future. All together 
35 innovative policy instruments were identified and analysed. The results of these analysis are presented the table 1 and in the following sub-chapters.

Table 1: Classification of the AGORA innovative policy instruments

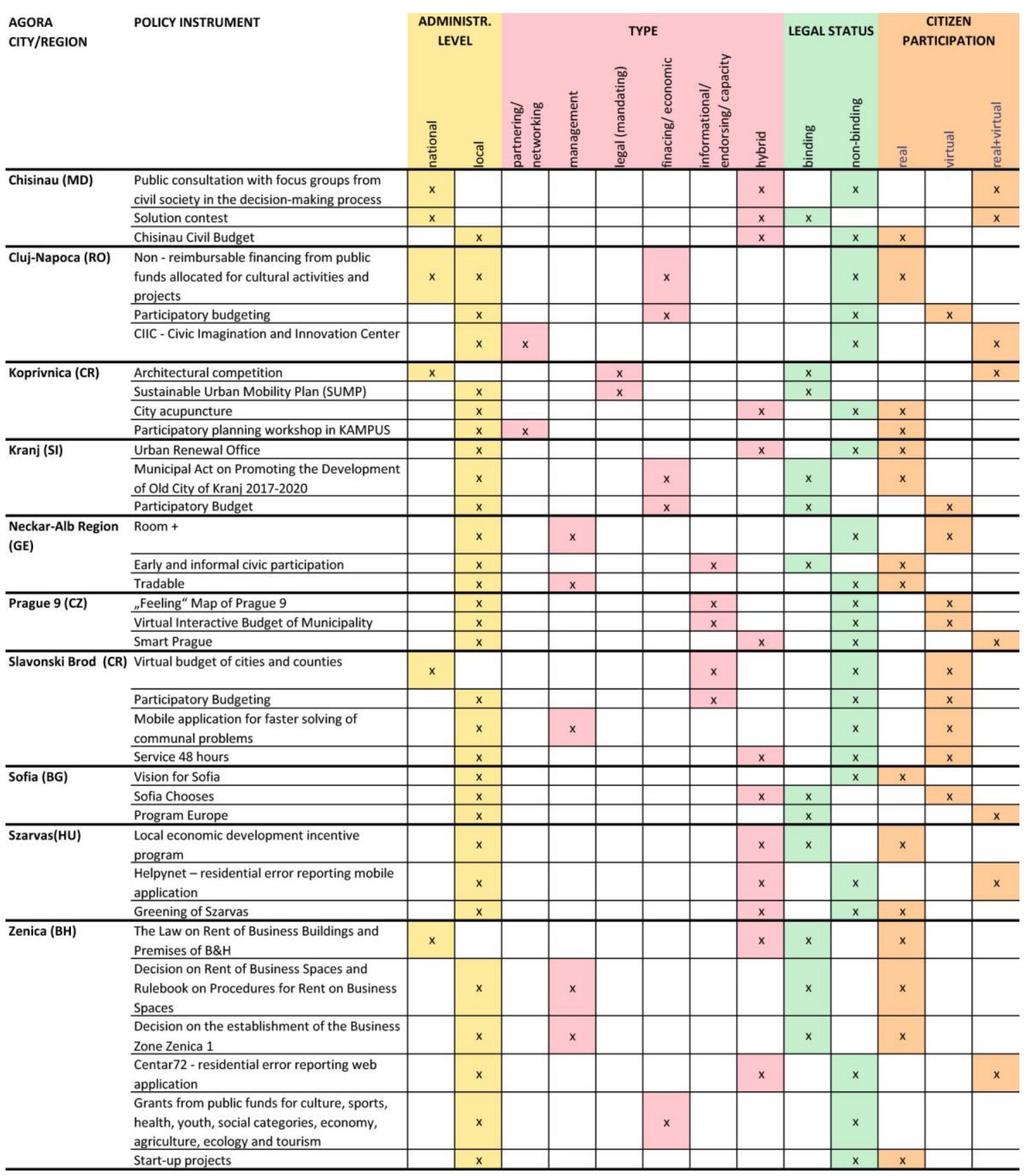

\subsection{Administrative level}

From the administrative point of view, given the focus of the implementation of urban regeneration endeavours, most cities provided many instruments from the local level, while indicating just few national administrative level instruments. On the national level, AGORA cities recognized five instruments (Chisinau, Koprivnica, Slavonski Brod and Zenica), while on the local level cities presented twenty-nine instruments altogether (see table 1). Only one policy instrument was recognised, both on the national and local level at the same time (Cluj-Napoca). 
The majority of identified instruments on the national level is related to the financial mechanisms that allow the urban regeneration processes to run more smoothly and faster (e.g. non-reimbursable funds, virtual budget, business-rents etc.). While the financial mechanisms are very much present on the local level too, they are largely complemented by the instruments that support the active participation of different stakeholders in the process (e.g. participatory planning workshops, informal participation etc.).

\subsection{Typology}

The innovative tools were further analysed regarding the main goals they were seeking to achieve. The analytical framework consisted of the six main categories:

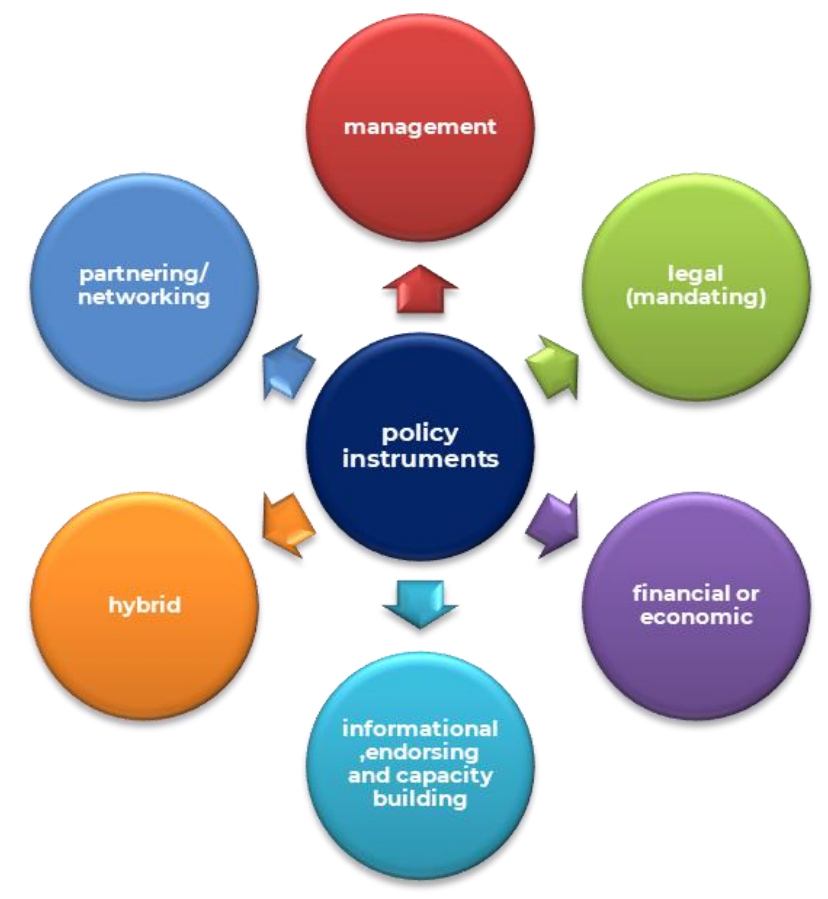

Figure 2 - Typology of policy instruments. Source: UIRS.

- partnering/networking instruments:

0 networks with other government bodies, public-private-partnerships (PPP), voluntary agreements, dialogues,

- organizational models with internal processes/workflow change in public administration (further used in the development of cross-departmental public administration task forces for AGORA),

- collaborative governance and join decision making models between private and public landowners.

- management instruments:

$\bigcirc$ as interaction interfaces for co-designing, co-implementing and co-management of urban assets in the forms of facilitated business to business (B2B), land-owners-tobusiness, governance-to-business (G2B) and governance to citizens (G2C),

- package schemes to assist private owners to identify partnership and funding options and overcome the current regulatory complexity for brownfield reuse.

- legal (mandating) instruments:

$\circ$ regulations, directives, laws, and decrees, 
○ procedural simplification (e.g. permitting), streamlining and fast-tracking procedures for revalorisation.

- financial or economic instruments:

- taxes, tax abatements, subsidies, and awards,

0 creative financing models for building, land recovery and reuse through new local value systems,

o other forms of collaborative funding.

- informational, endorsing and capacity building instruments:

- websites, brochures, campaigns, guidelines, trainings, conferences, or labels,

- new methodologies for consolidating capacity building and technical expertise in public administration and

- hybrid instruments:

○ i.e. strategies or action plans, platforms.

The analyses showed that the most of the innovative policy instruments listed by the AGORA partners could be classified in more than one of the above presented categories, i.e. they are distinctively hybrid in their nature and goals (trying to address and achieve more than one main goal). Besides the innovative instruments that are hybrid, most of those instruments falls under "Financial/economic" and "Informational/endorsing/capacity building" categories (see table 1).

\subsection{Legal status}

Among identified innovative urban regeneration instruments, nearly two thirds of them, were nonebinding (see table 1) meaning that they complement the legally binding procedures. An interesting observation is that there are no clear distinctions between biding and none-binding tools among the partners - in some cases or for some partners the usage of the similar tool can be biding while the same tool might be none-binding in the case of another partner (e.g. participatory budgeting).

\subsection{Citizen participation}

Innovative policy instruments have been analysed regarding the type of participation of the actors too within two main categories: participation in person (real participation - when participation in concrete location and place is required or is mandatory for the instrument to function) and virtual (in cases where the participation is required to be largely or entirely online). In some other cases, innovative policy instruments can require both real and virtual participation. This analytical aspect got even more important under the covid-19 pandemics limitations to the public life, it gave the understanding of the ability of the innovative tool to (not) support urban regeneration during the restrictions of the public life.

Altogether cities presented 15 innovative policy instruments requiring real participation and 9 policy instruments requiring virtual participation; moreover 9 policy instruments cannot be identified in just one of these two categories as they imply the usage of both types of participation (see the table 1).

\section{Discussion and conclusions}

There are few major observations that can be done after the review and analyses of the innovative urban regeneration instruments within the AGORA project which is running in the Danube Region.

The analyses revealed that the notion of "innovative" differs largely across the region. This does not come as a surprise if the innovation is understood as a step into a new direction and away from doing business as usual - some practices that have been adopted by some partners long ago are seen as a complete novelty for some other partners and/or places and regarded as innovative. This reveals the 
existing gap in the level of exchanges and connectedness within the region when it comes to the usage of the instruments for the more efficient implementation of urban regeneration interventions.

Nevertheless, there seems to be a large consensus across the region that some of identified instruments can be generally labelled as innovative. Such examples include among others:

- the participatory budgeting; different official names are given to these kinds of the instruments (e.g. Participatory budgeting, Civil budget, Virtual budget, etc.), but the main aim is the same - to give the voice and decision-making power on public money expenditure to the final users - the citizens,

- none-reimbursable financing instruments: these include grants from public funds for various activities and programmes (e.g. culture, sport, youth, healthy lifestyles etc.) as well as development of businesses (including start-ups and incubators support, rents for business premises etc.),

- instruments to strengthen the communities: in a real or virtual format; these innovative instruments aim to empower the local communities via cooperation and co-creation activities which can help to generate the process of urban regeneration (e.g. urban renewal offices, civic imagination \& innovation centre etc.); rather often an important dimension is the co-generation of common ideas where the process is sometimes more important than the idea itself as it strengthens the relations and cooperation capacity of the local community,

- instruments to improve the citizen - authority communication and cooperation; these instruments aim to reach beyond the information sharing practice and try to establish a meaningful relationship between the local authorities and citizens; the early stage of such endeavours are e.g. the web-portals where the citizens are in the role of sensors and report the needed maintenance works on the public infrastructures, but can be later upgraded to the formats that allow citizens to express their ideas and visions on the future development and seek the feedback from the authorities; in an ideal form these instruments also reach the level of a common decision-making, where relevant issues are discussed among citizens and authorities (e.g. focus groups) and solutions tested together (e.g. urban acupuncture, participatory planning workshops etc.).

It can also be noted that the new, innovative instruments in urban regeneration are mostly introduced at the local level, while the innovation at the intermediate and/or national levels are less likely. This observation is expected as the urban planning and related procedures are usually the responsibility of the local administrations. On one hand this approves the need for the local planning administration to have as open hands as possible when deciding its urban development and regeneration strategies in order to be able to introduce innovative solutions tailored to the needs of their concrete context. On the other hand, it puts the light on the need for the wider frameworks that will transcend the local levels and introduce innovation at the upper administrative levels too, e.g. regional and (trans)national level. AGORA project is the step in this direction as it makes the review of the state of the art and meaningful exchanges of knowledge and practices in the field of urban regeneration among different local administrations and thus contribute to the foundations of the possible new innovative approaches at the regional and European levels too.

Acknowledgement: This research was partly funded by the AGORA project which is co-financed by the INTERREG Danube Programme.

\section{References}

AGORA Resources 
Mušič, B., et al. (2021). Deliverable 'D.T1.2.1 Guidance on the operational analysis in partner cities'. AGORA. Interreg Programme, Project Code DTP3-353-4.1, European Commission

Mušič, B., et al. (2021). Deliverable 'D.T1.3.1 Framework for assessment and benchmarking'. AGORA. Interreg Programme, Project Code DTP3-353-4.1, European Commission

Mušič, B., Jurca, T., (2021). Deliverable 'D.T1.3.2 Best practice report'. AGORA. Interreg Programme, Project Code DTP3-353-4.1, European Commission

External references

Cirera, X., Jaime, F., Justin, H., Yanchao, L. (2020). 'A Practitioner's Guide to Innovation Policy : Instruments to Build Firm Capabilities and Accelerate Technological Catch-Up in Developing Countries'. World Bank, Washington, DC. (C) World Bank. https://openknowledge.worldbank.org/handle/10986/33269

Edquist, C. (2011). 'Design of Innovation Policy through Diagnostic Analysis: Identification of Systemic Problems (or Failures).' Industrial and Corporate Change 20 (6): 1-29.

Freeman, C. (1987). 'Technology Policy and Economic Performance: Lessons from Japan'. London: Pinter

Howlett, M., Rayner, J. (2007) 'Design Principles for Policy Mixes: Cohesion and Coherence in 'New Governance Arrangements', Policy and Society, 26:4, 1-18. Available at: DOI 10.1016/S1449 4035(07)70118-2

Lascoumes, P. and Le Gales, P. (2007). 'Introduction: Understanding Public Policy through Its Instruments -From the Nature of Instruments to the Sociology of Public Policy Instrumentation'. Governance, 20: 1-21. https://doi.org/10.1111/j.1468-0491.2007.00342.x

Majoor S., Schwartz K. (2015) Instruments of Urban Governance. In: Gupta J., Pfeffer K., Verrest H., RosTonen M. (eds) Geographies of Urban Governance. Springer, Cham. https://doi.org/10.1007/9783-319-21272-2_6

Schneider, A., Ingram, H. (1990) 'Behavioural assumptions of policy tools'. J Polit 52(2):510-529 